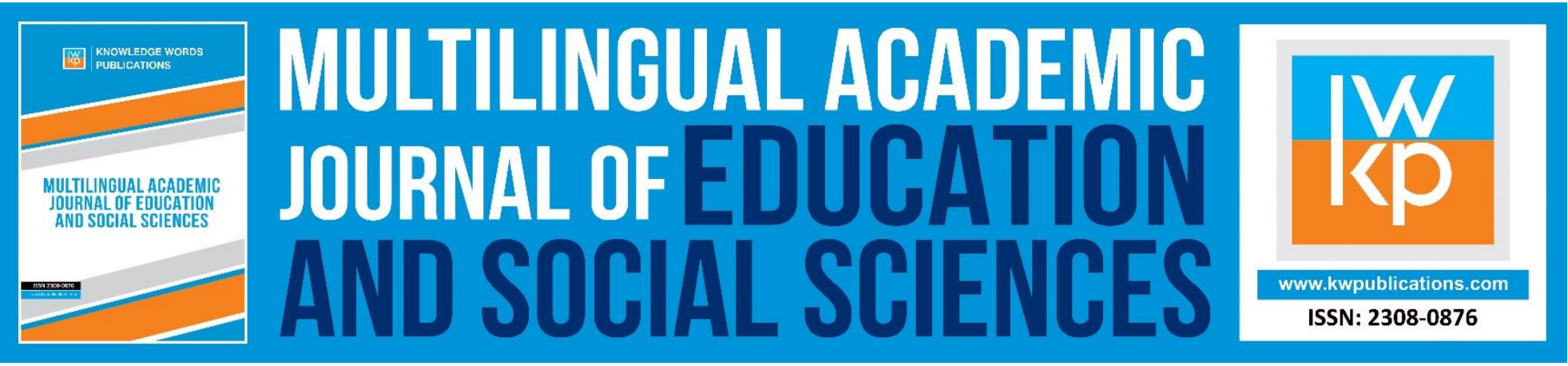

\title{
Creative Writing, Digital Storytelling and the Arts in EFL: The Case of Storybird
}

Kyrmanidou Elli

To Link this Article: http://dx.doi.org/10.46886/MAJESS/v8-i1/6971

DOI:10.46886/MAJESS/v8-i1/6971

Received: 02 June 2020, Revised: 09 July 2020, Accepted: 17 August 2020

Published Online: 25 September 2020

In-Text Citation: (Kyrmanidou, 2020)

To Cite this Article: Kyrmanidou E. (2020). Creative Writing, Digital Storytelling and the Arts in EFL: The Case of Storybird. Multilingual Academic Journal of Education and Social Sciences, 8(1), 66-75 (in Greek).

Copyright: ( ) The Authors 2020

Published by Knowledge Words Publications (www.kwpublications.com)

This article is published under the Creative Commons Attribution (CC BY 4.0) license. Anyone may reproduce, distribute, translate and create derivative works of this article (for both commercial and non-commercial purposes), subject to full attribution to the original publication and authors. The full terms of this license may be seen

at: http://creativecommons.org/licences/by/4.0/legalcode

Vol. 8, No. 1, 2020, Pg. 66 - 75

https://kwpublications.com/journals/journaldetail/MAJESS

JOURNAL HOMEPAGE

Full Terms \& Conditions of access and use can be found at https://kwpublications.com/pages/detail/publication-ethics 


\title{
Creative Writing, Digital Storytelling and the Arts in EFL: The Case of Storybird
}

\author{
Kyrmanidou Elli \\ Ludwig Maximilian University of Munich
}

\begin{abstract}
In the context of foreign language education new technologies have transformed traditional teaching into a learning experience where students make use of a variety of digital tools in order to create authentic multimodal texts. The new digital environments should not, however, replace the students' fundamental cognitive skills but promote the basic principles of the humanities combined with digital technology. This will contribute to a holistic approach of foreign language teaching and encourage multiliteracy. The aim of the following paper is to explore the various advantages of the digital tool Storybird which enables the implementation of the pedagogical principles of creative writing, digital storytelling and the contribution of the Arts in the learning procedure. Creative writing disengages the foreign language teaching from the passive transfer of knowledge and engages it with the students' imagination and creativity. Digital storytelling reinforces the interactive nature of foreign language teaching and transforms the target language into a tool for digital communication. The pictures in Storybird facilitate the connection between the classroom and the Arts and cultivate the students' aesthetic experience promoting differentiated instruction in EFL
\end{abstract}

Keywords: EFL, Creative Writing, Digital Storytelling, Arts, Storybird.

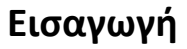

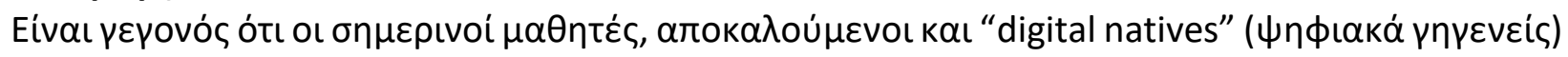

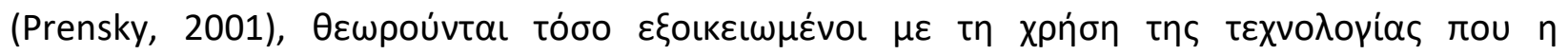

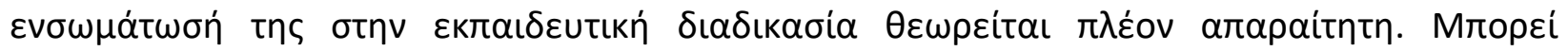

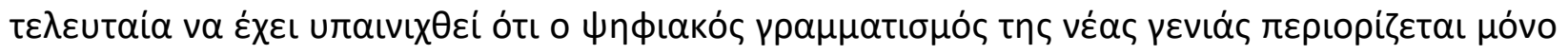

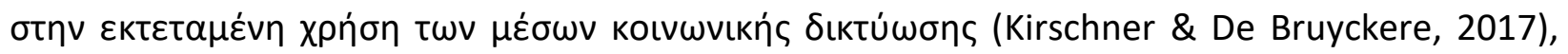

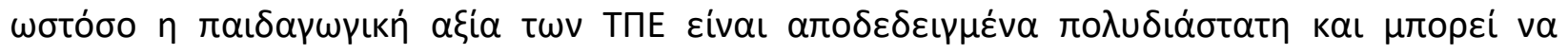

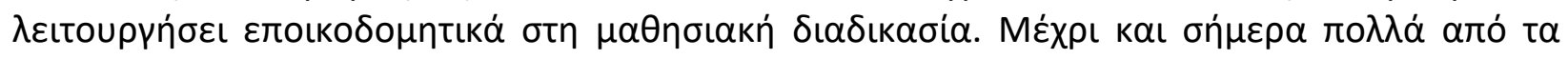

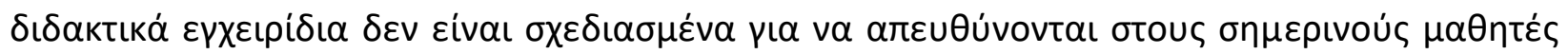

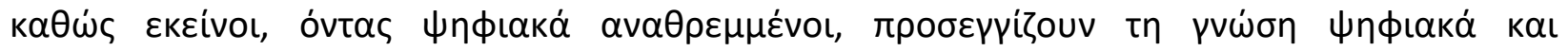

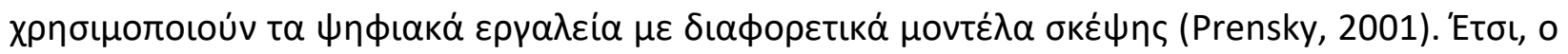

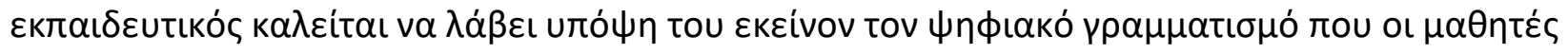


MULTILINGUAL ACADEMIC JOURNAL OF EDUCATION AND SOCIAL SCIENCES

Vol. 8 No. 1, 2020, E-ISSN: 2308-0876 @ 2020 KWP

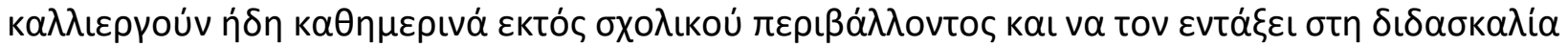

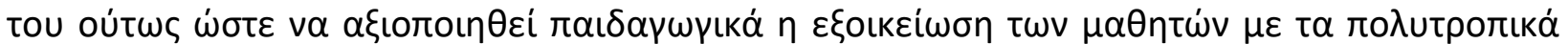

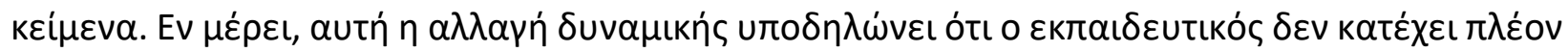

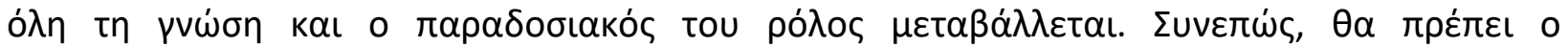

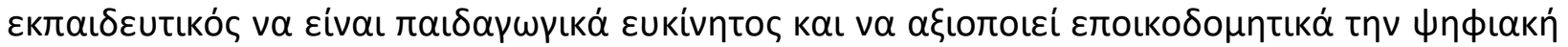

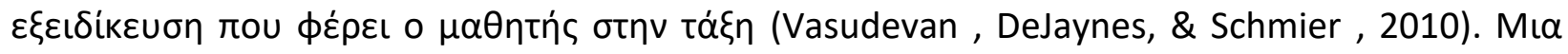

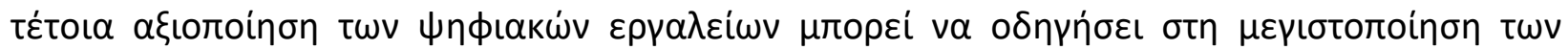

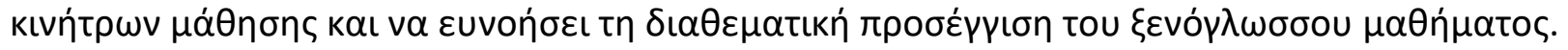

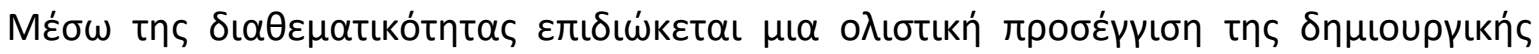

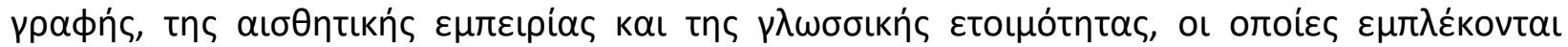

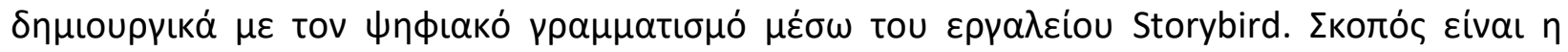

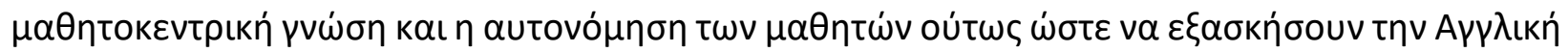

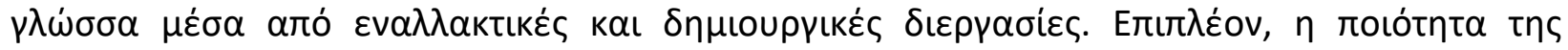

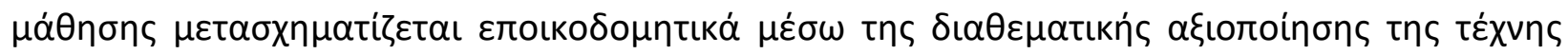

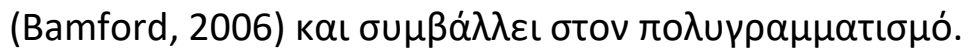

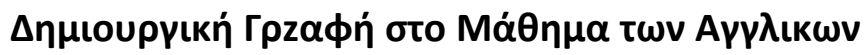

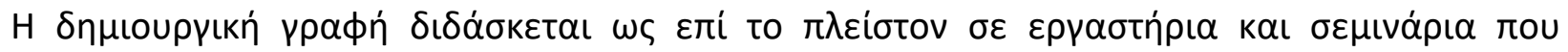

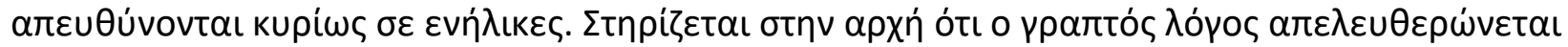

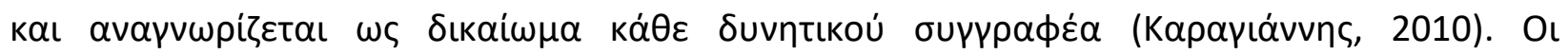

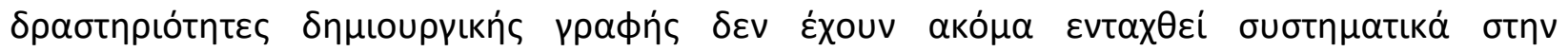

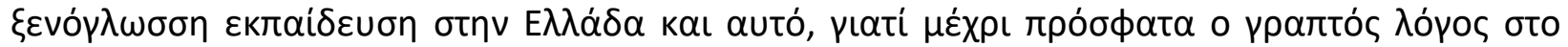

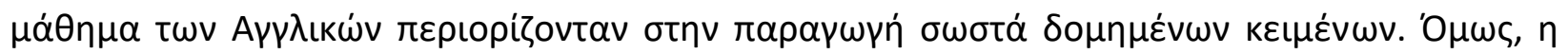

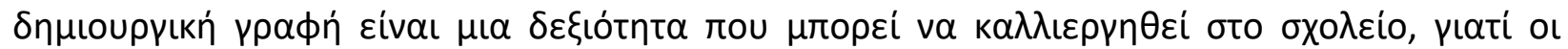

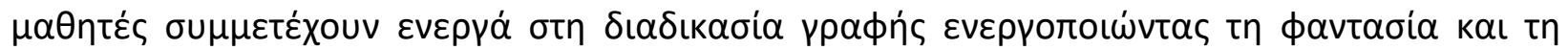

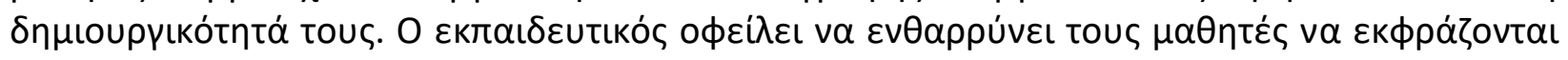

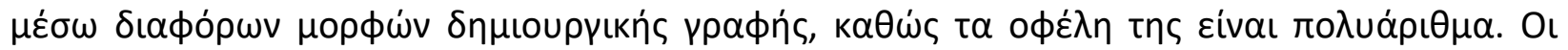

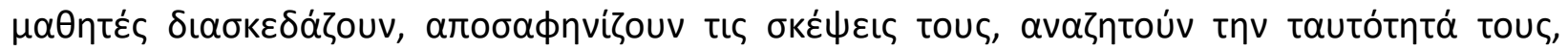

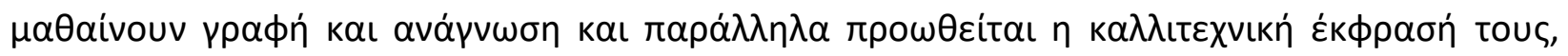

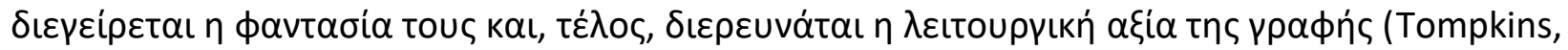

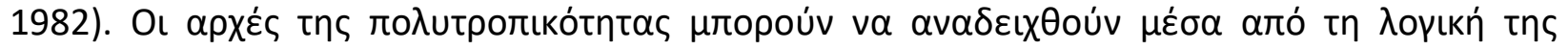

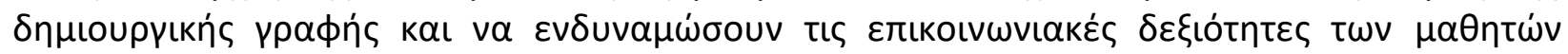

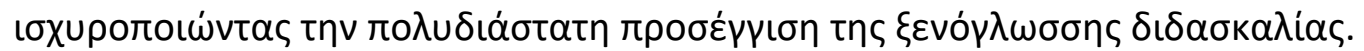

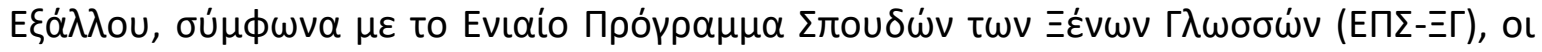

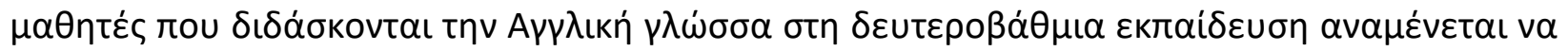

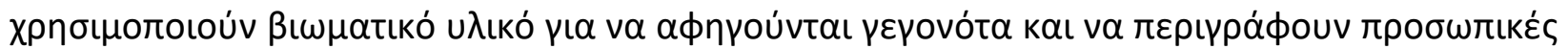

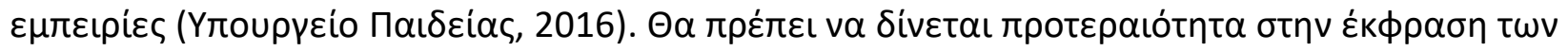

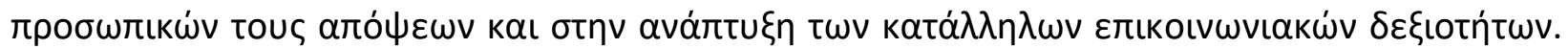

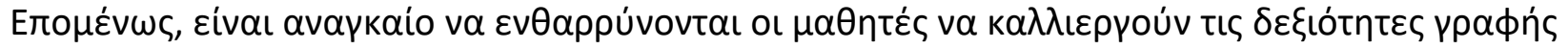

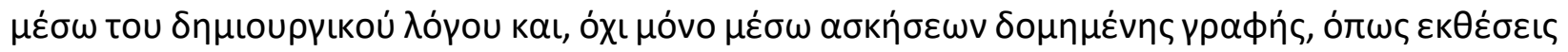

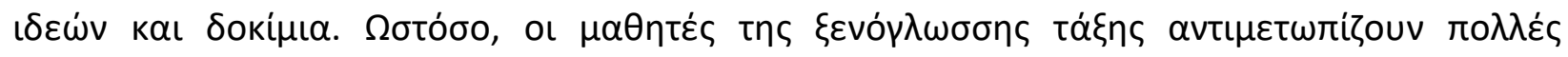

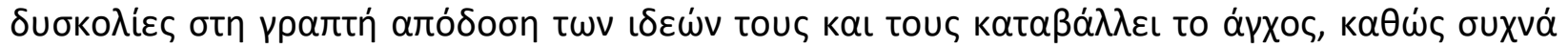


MULTILINGUAL ACADEMIC JOURNAL OF EDUCATION AND SOCIAL SCIENCES

Vol. 8 No. 1, 2020, E-ISSN: 2308-0876 @ 2020 KWP

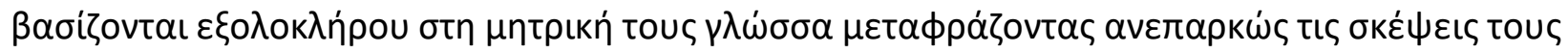

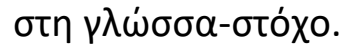

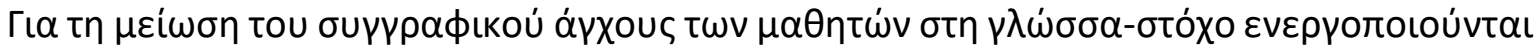

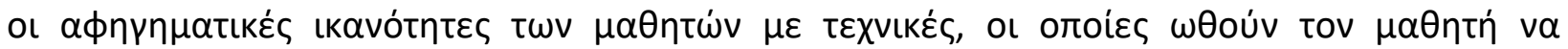

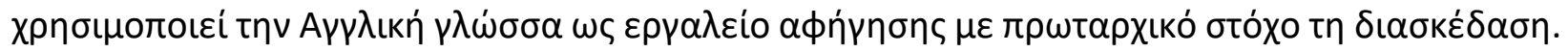

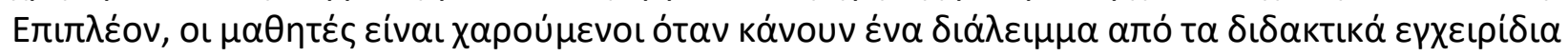

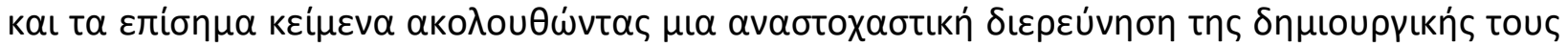

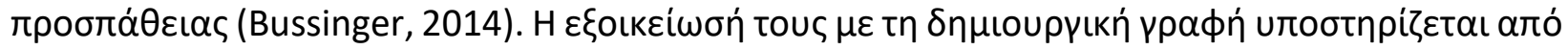

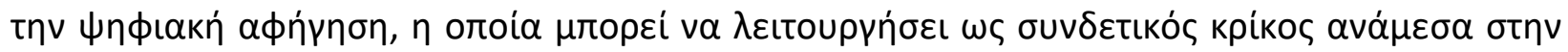

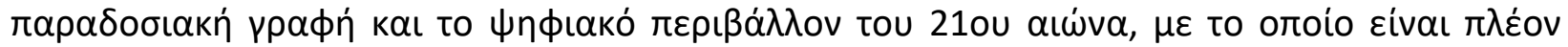

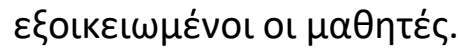

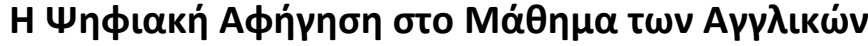

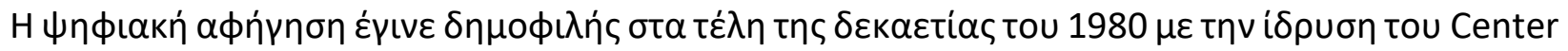

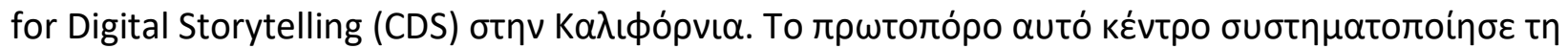

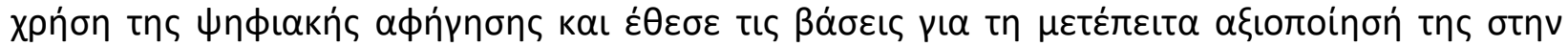

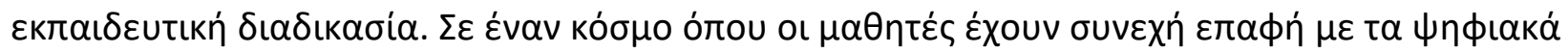

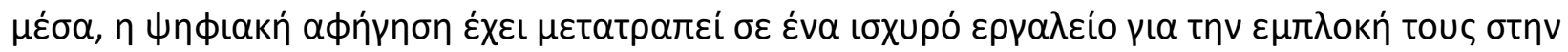

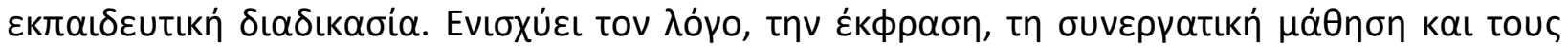

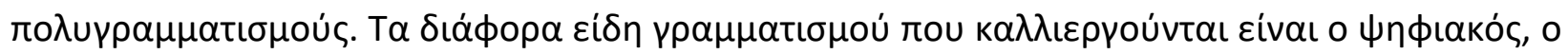

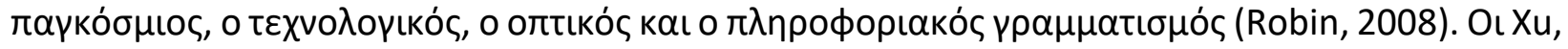

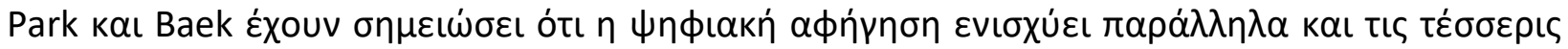

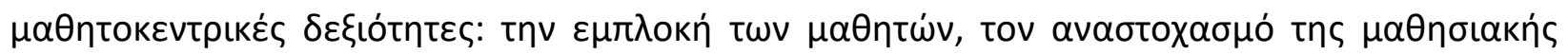

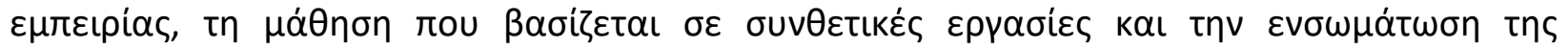

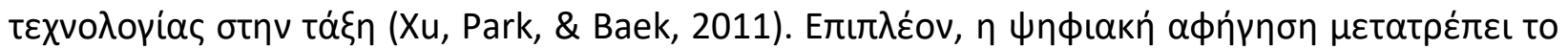

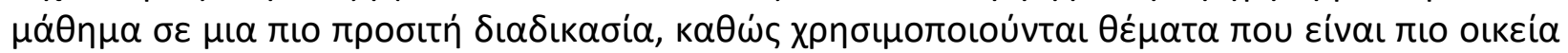

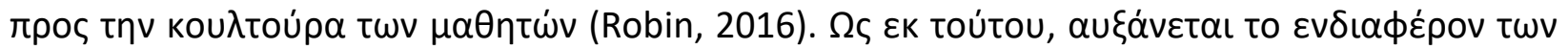

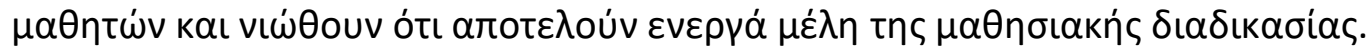

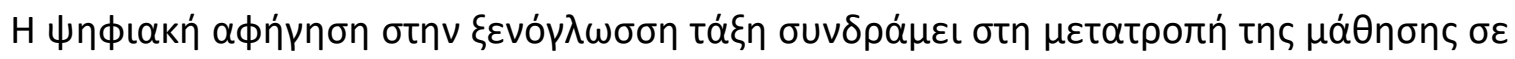

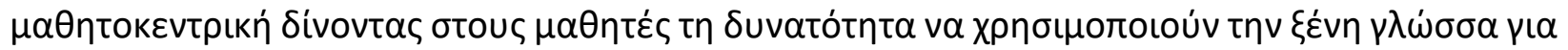

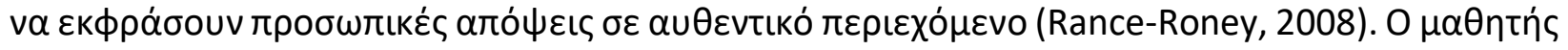

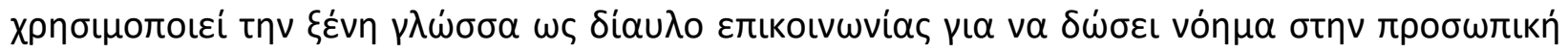

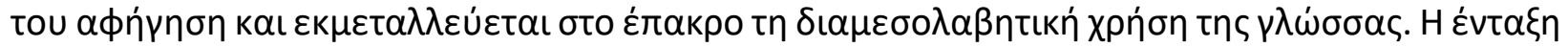

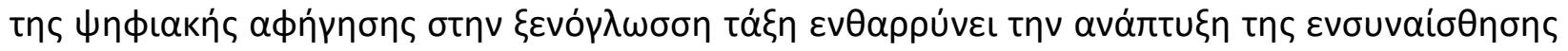

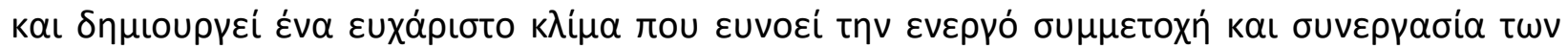

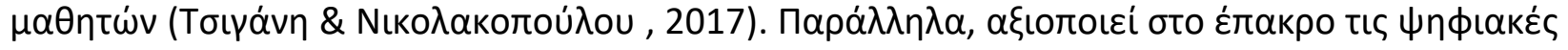

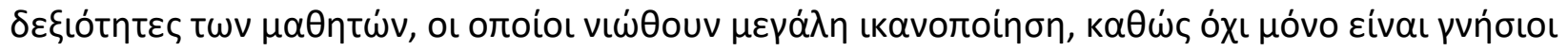

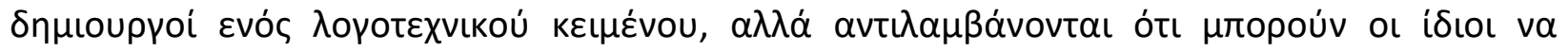

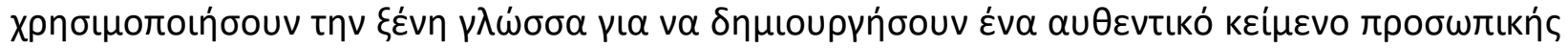

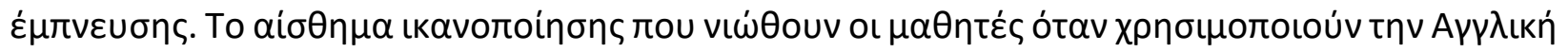

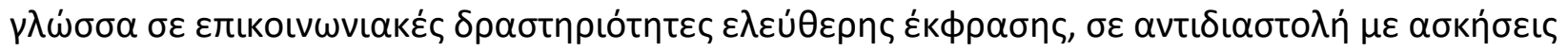

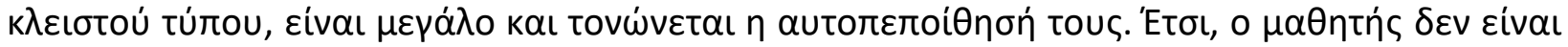

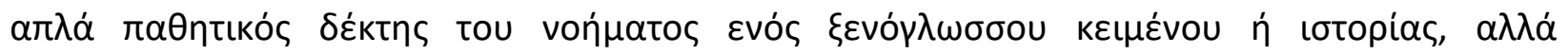


MULTILINGUAL ACADEMIC JOURNAL OF EDUCATION AND SOCIAL SCIENCES

Vol. 8 No. 1, 2020, E-ISSN: 2308-0876 @ 2020 KWP

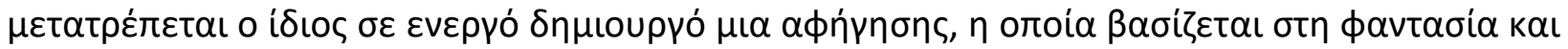

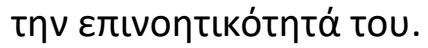

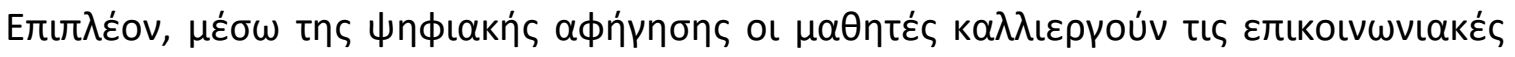

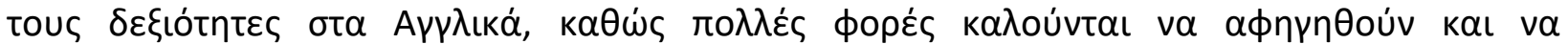

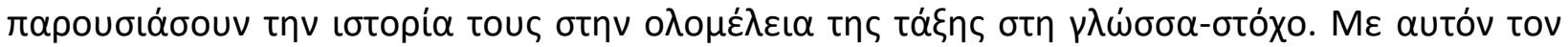

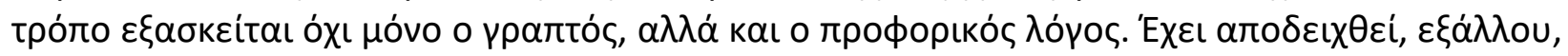

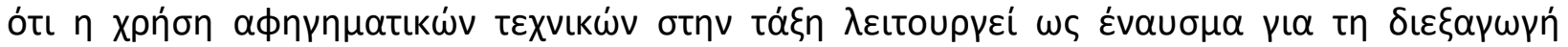

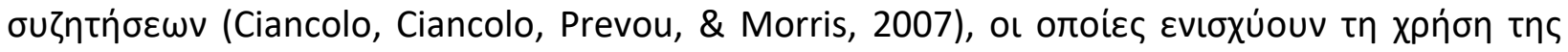

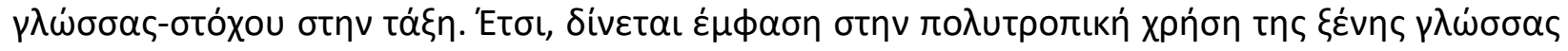
(

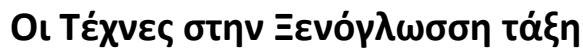

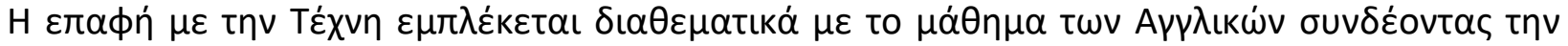

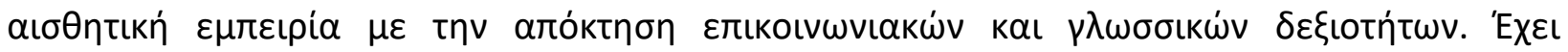

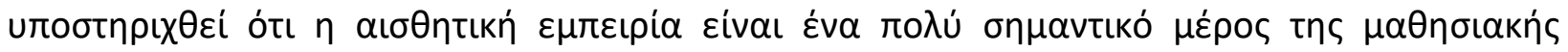

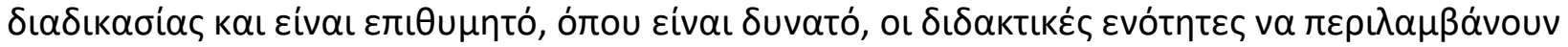

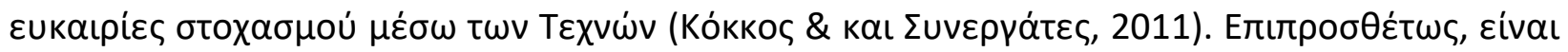

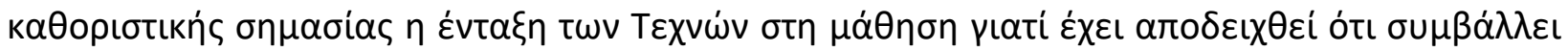

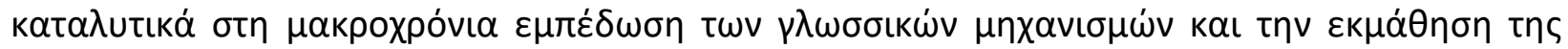

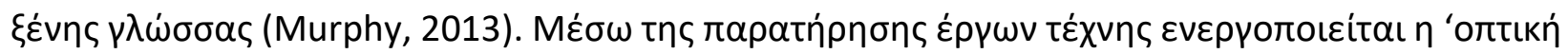

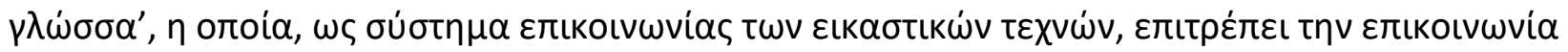

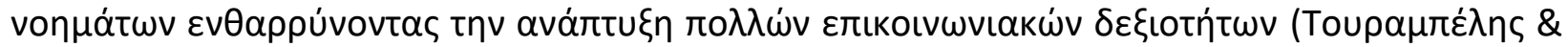

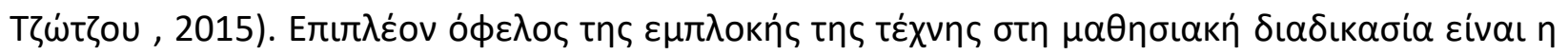

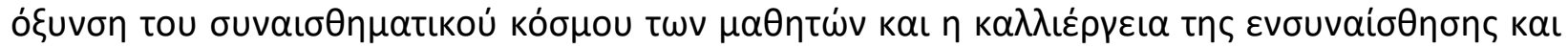

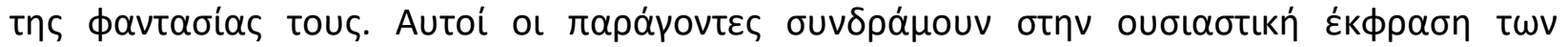

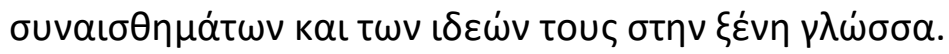

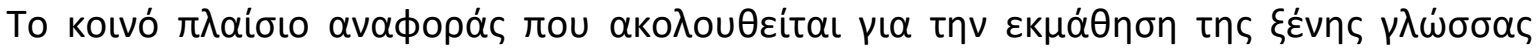

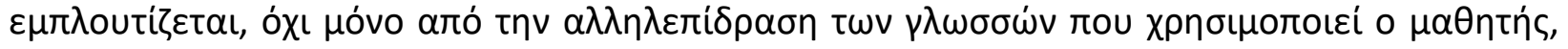

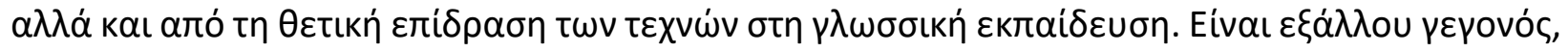

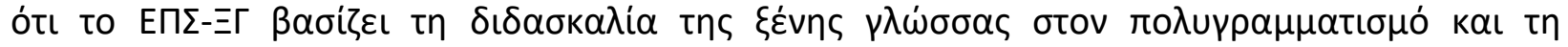

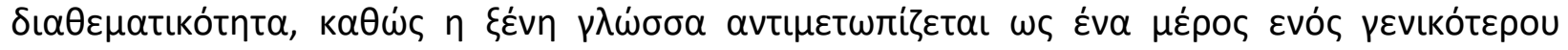

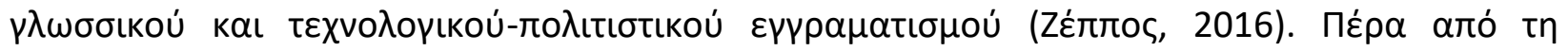

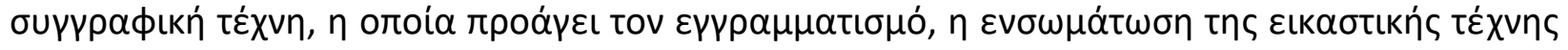

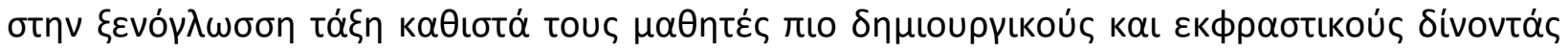

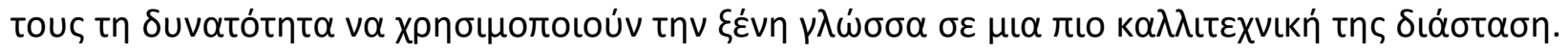

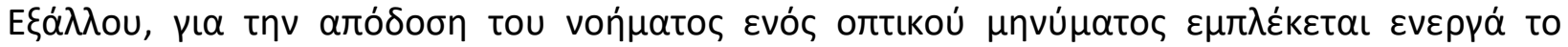

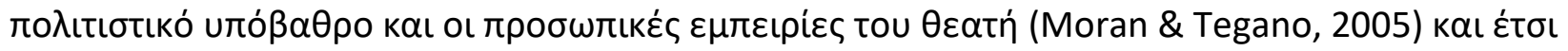

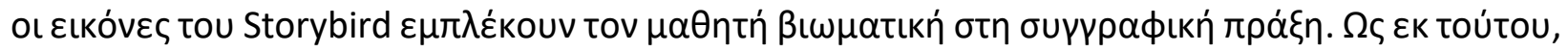

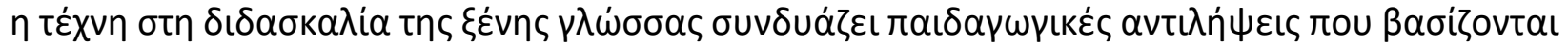


MULTILINGUAL ACADEMIC JOURNAL OF EDUCATION AND SOCIAL SCIENCES

Vol. 8 No. 1, 2020, E-ISSN: 2308-0876 @ 2020 KWP

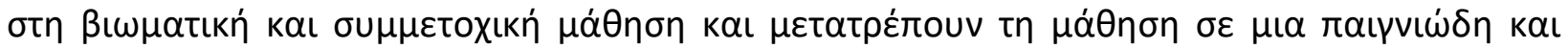

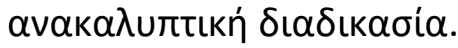

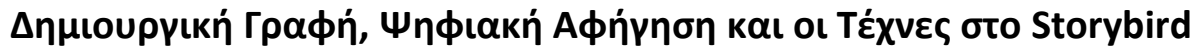

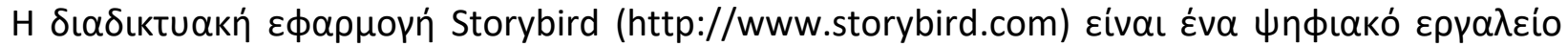

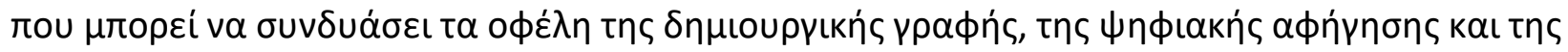

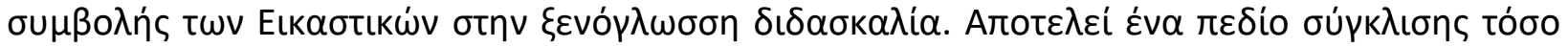

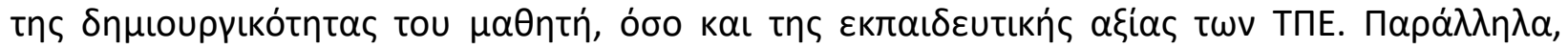

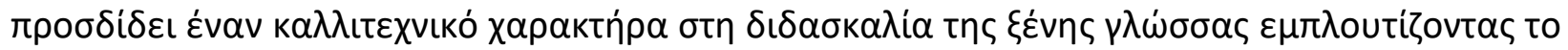

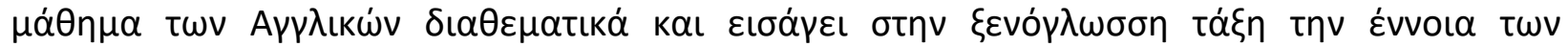

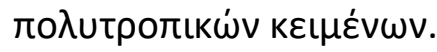

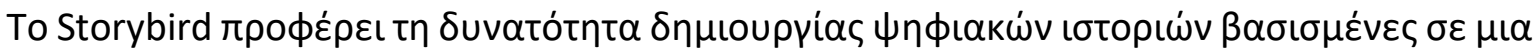

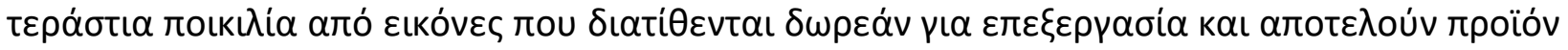

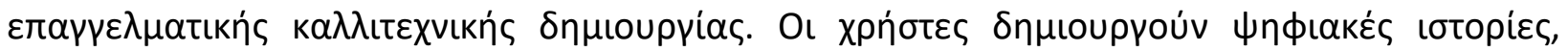

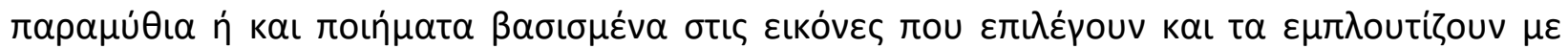

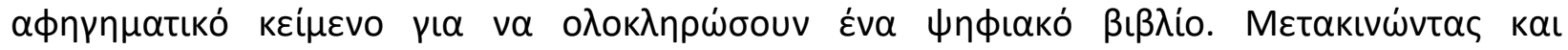

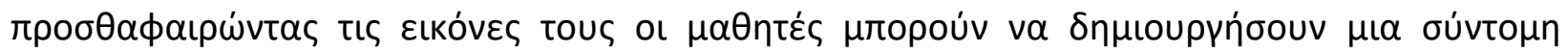

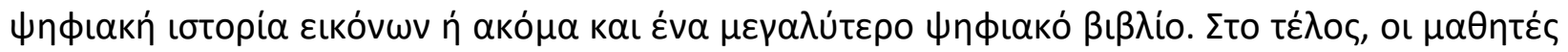

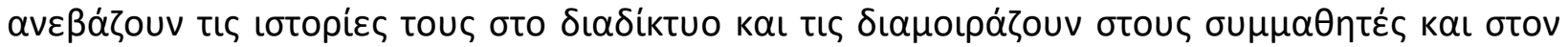

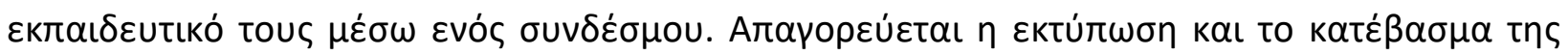

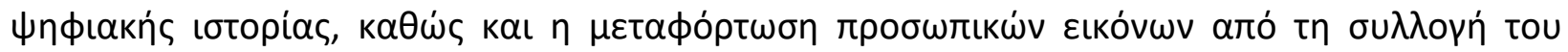

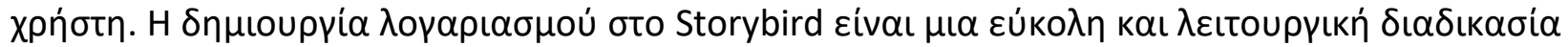

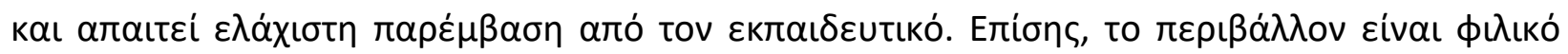

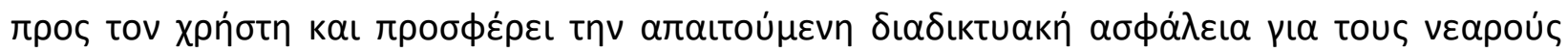

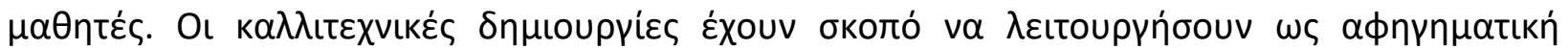

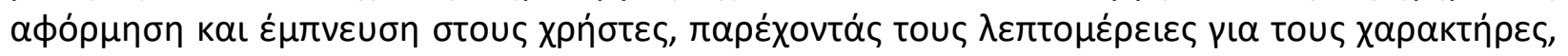

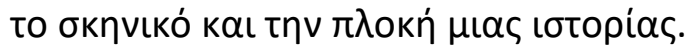

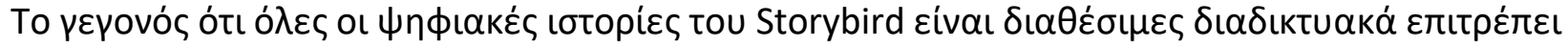

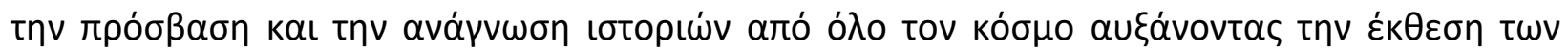

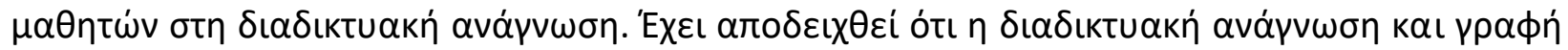

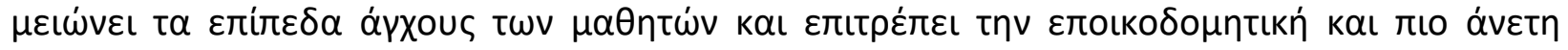

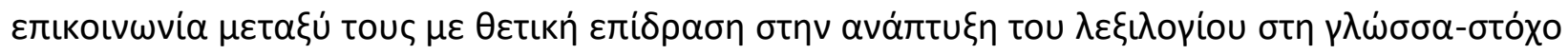

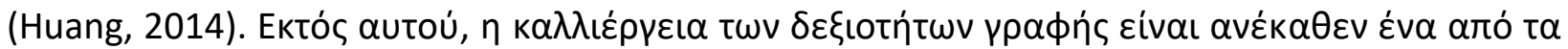

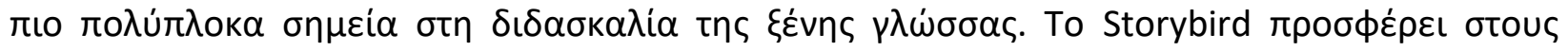

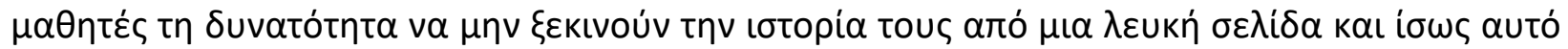

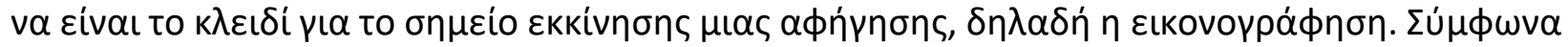

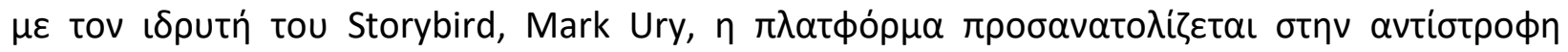

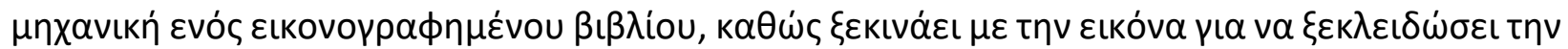

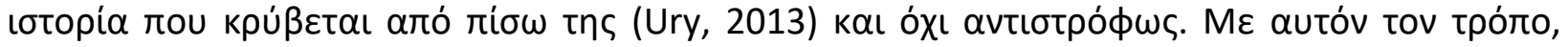

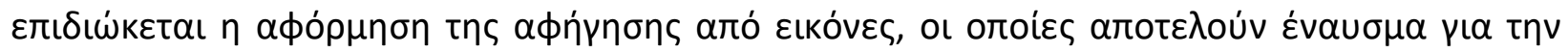

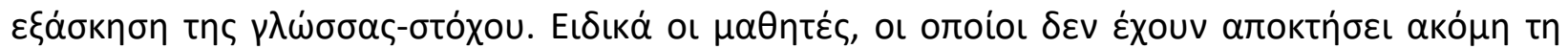

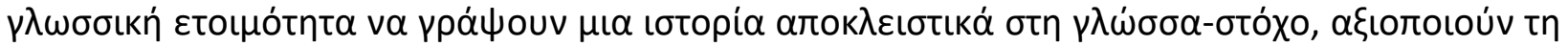

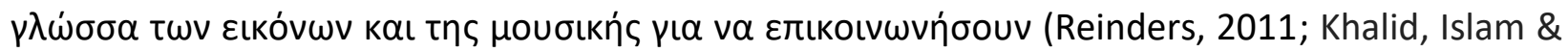
Ahmed, 2019; Alzgool, 2019; Umrani, Ahmed \& Memon, 2015; Zin \& Ibrahim, 2020). To Storybird 
MULTILINGUAL ACADEMIC JOURNAL OF EDUCATION AND SOCIAL SCIENCES

Vol. 8 No. 1, 2020, E-ISSN: 2308-0876 @ 2020 KWP

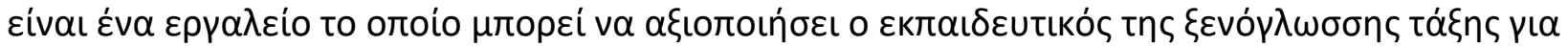

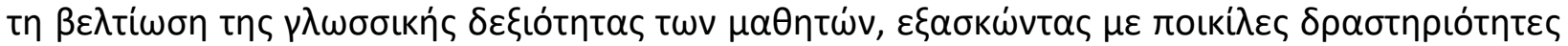

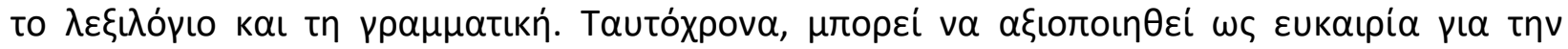

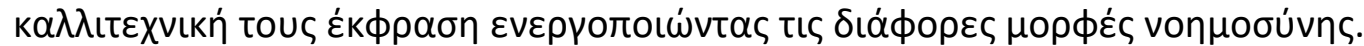

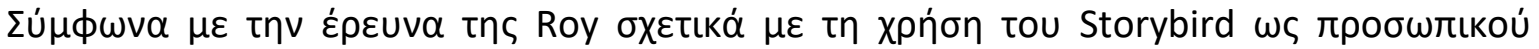

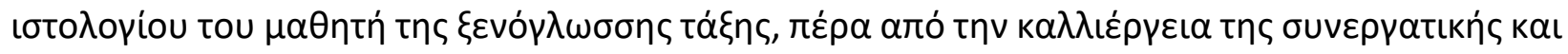

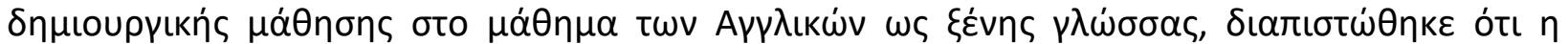

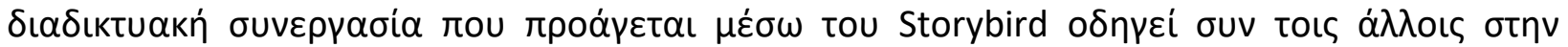

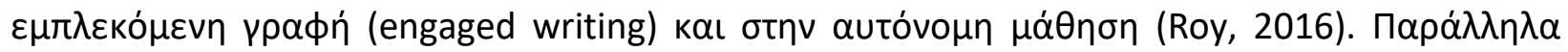

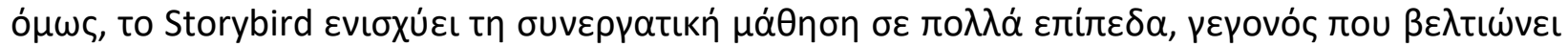

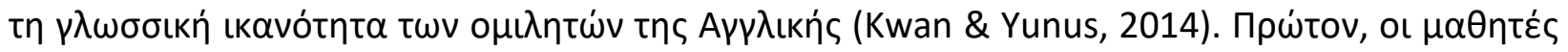

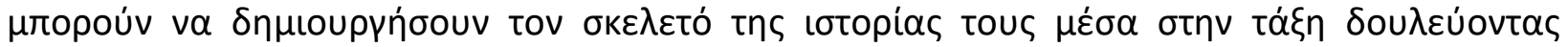

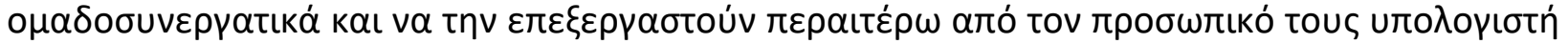

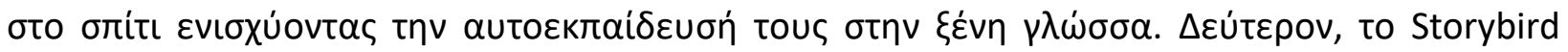

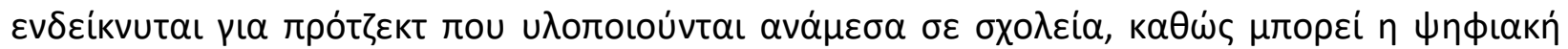

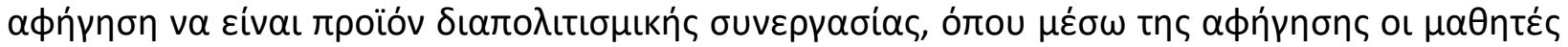

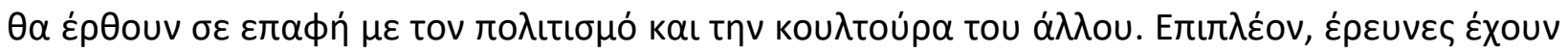

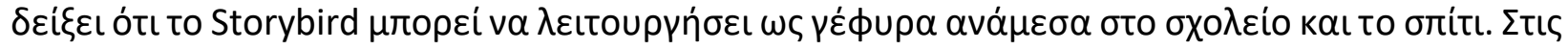

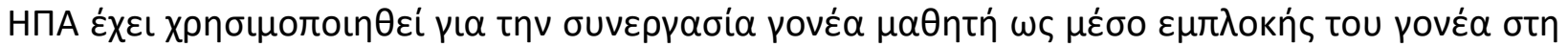

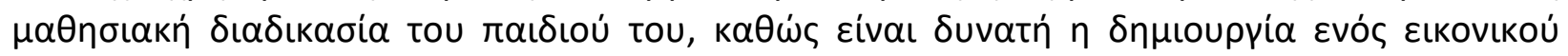

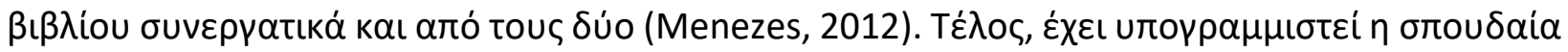

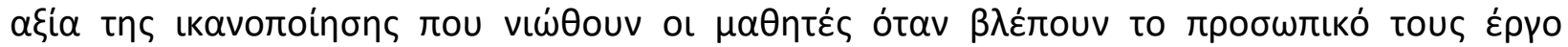

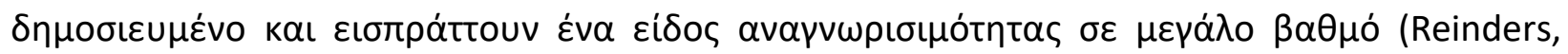
2011).

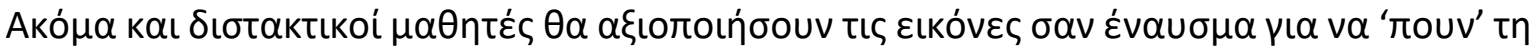

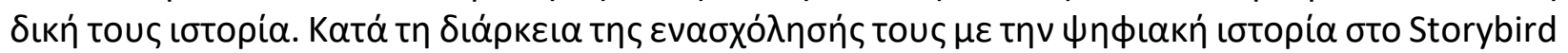

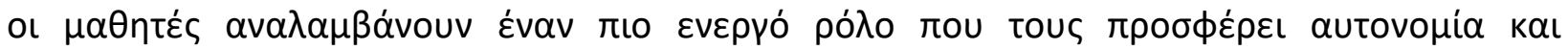

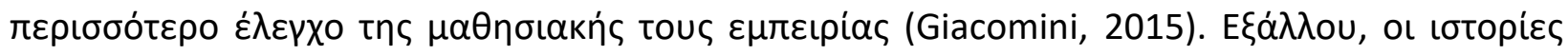

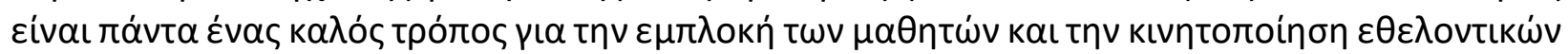

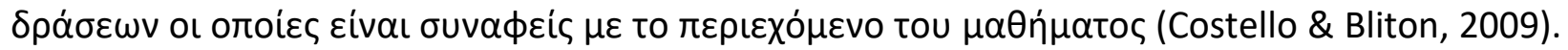

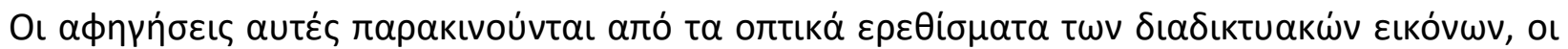

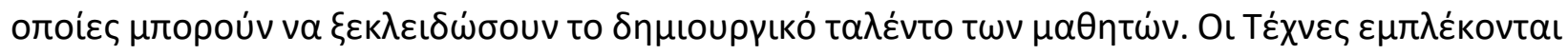

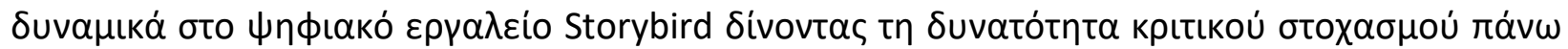

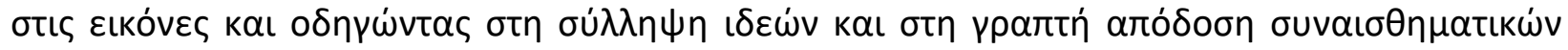

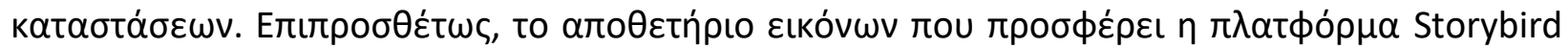

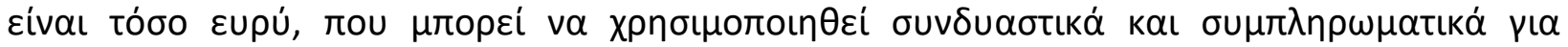

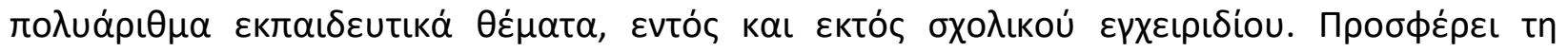

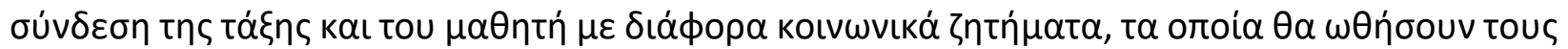

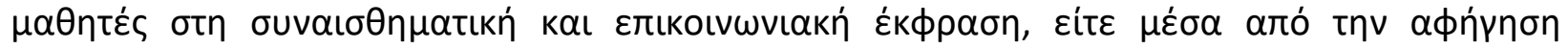

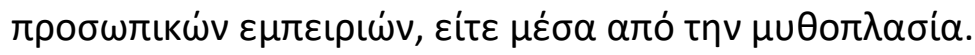

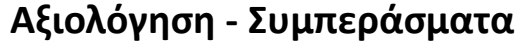

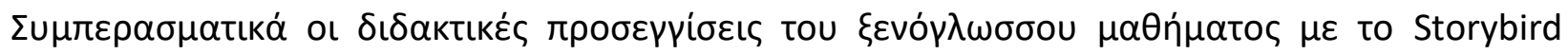

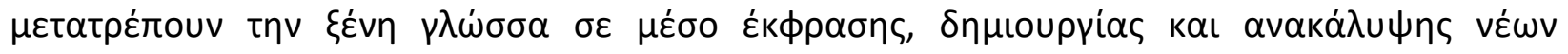


MULTILINGUAL ACADEMIC JOURNAL OF EDUCATION AND SOCIAL SCIENCES

Vol. 8 No. 1, 2020, E-ISSN: 2308-0876 @ 2020 KWP

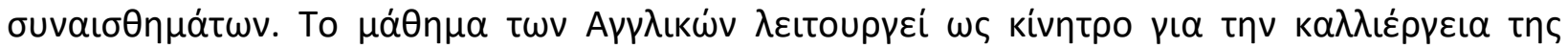

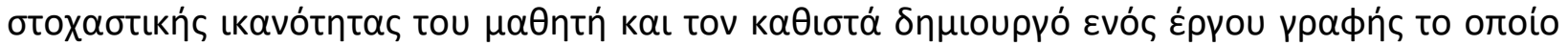

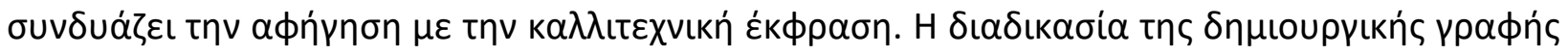

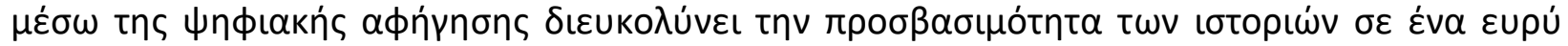

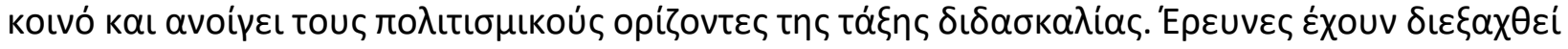

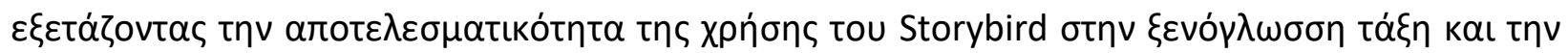

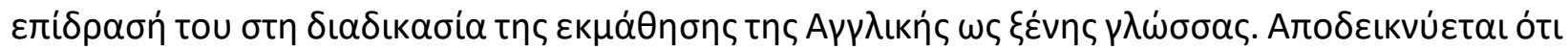

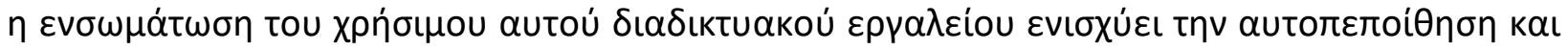

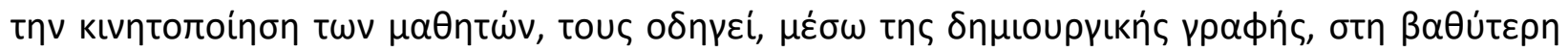

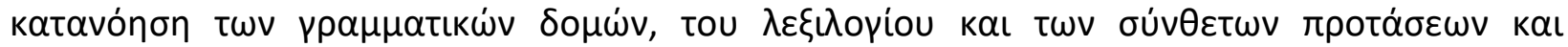

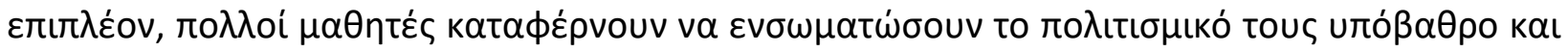

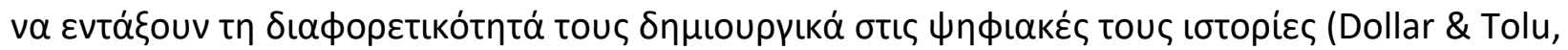

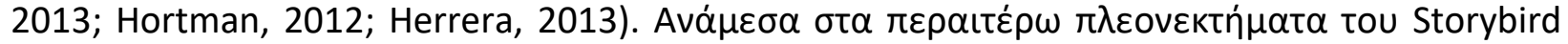

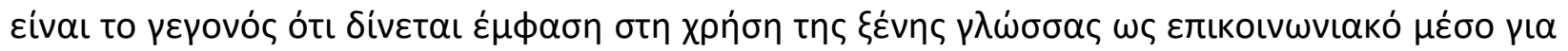

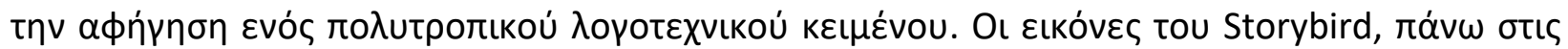

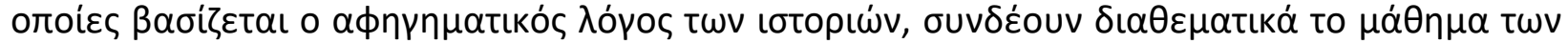

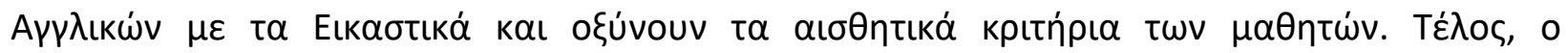

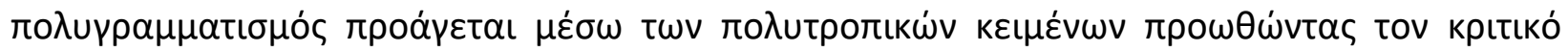

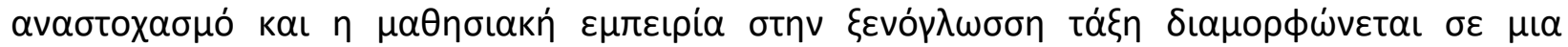

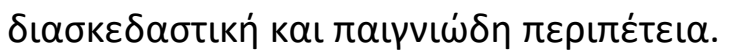

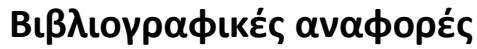 \\ References}

Alzgool, M. (2019). Nexus between green HRM and green management towards fostering green values. Management Science Letters, 9(12), 2073-2082.

Bamford, A. (2006). The wow factor: global research compendium on the impact of the arts in education. New York: Waxmann.

Bussinger, C. R. (2014). Creative writing in ELT: Organically grown stories. The Journal of Literature in Language Teaching, 2(2), 12-18.

Ciancolo, A. T., Ciancolo, D., Prevou, M. I., \& Morris, I. F. (2007). Using digital storytelling to stimulate discussion in Army professional forums. Proceedings of the Interservice/Industry Training, Education, \& Simulation Conference (I/ITSEC).

Costello, R., \& Bliton, D. (2009). Assessment of Educational Visual Storytelling at the Smithsonian.

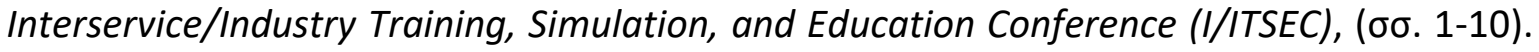
Paper No 9445.

Dollar, Y. K., \& Tolu, A. T. (2013). Examining the 5th Grade English Language Learners' First Experience of Writing Digital Stories: A Case Study. Proceedings of SITE 2013--Society for Information Technology \& Teacher Education International Conference (pp. 16731677). New Orleans, Louisiana: Association for the Advancement of Computing in Education (AACE).

Giacomini, L. (2015). Using "Storybird" in Young Learners' Creative Writing Class. English Teaching

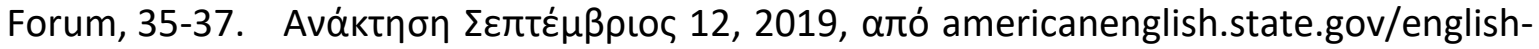
teaching-forum 
MULTILINGUAL ACADEMIC JOURNAL OF EDUCATION AND SOCIAL SCIENCES

Vol. 8 No. 1, 2020, E-ISSN: 2308-0876 @ 2020 KWP

Herrera, Y. E. (2013). Writing skill enhancement when creating narrative texts through the use of collaborative writing and the Storybird Web 2.0 tool. Colombian Applied Linguistics Journal, 15(2), 166-183.

Hortman, A. (2012). ELL Writing Strategies: A Case Study. Kennesaw State.

Huang, H.-C. (2014). Online Versus Paper-based Instruction: Comparing Two Strategy Training Modulesfor Improving Reading Comprehension. RELC Journal, 45(2), 165-180.

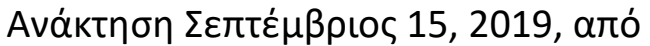
https://journals.sagepub.com/doi/10.1177/0033688214534797

Khalid, N., Islam, D. M. Z., \& Ahmed, M. R. M. (2019). SENTREPRENEURIAL TRAINING AND ORGANIZATIONAL PERFORMANCE: IMPLICATIONS FOR FUTURE. Humanities \& Social Sciences Reviews, 7(2), 590-593.

Kirschner, P. A., \& De Bruyckere, P. (2017). The myths of the digital native and the multitasker. Teaching and Teacher Education, 135-142.

Kwan, L. S., \& Yunus, M. M. (2014). Group Participation and Interaction in ESL Wiki Collaborative Writing among Malaysian Gifted Students. Asian Social Science, 11(59).

Menezes, H. (2012). Using Digital Storytelling to Improve Literacy Skills. IADIS International Conference on Cognition and Exploratory Learning in Digital Age (CELDA 2012). Madrid.

Moran, M. J., \& Tegano D. W., (2005). Moving toward Visual Literacy: Photography as a Language of Teacher Inquiry. Early Childhood Research and Practice, 7(1).

Murphy, A. F. (2014). Integrating Aesthetics into Professional Development for Teachers of English Learners. TESOL, 5(1), 82-104.

Prensky, M. (2001). Digital Natives, Digital Immigrants Part 1. On the Horizon, 9, 1-6. doi:http://dx.doi.org/10.1108/10748120110424816

Rance-Roney, J. (2008). Digital storytelling for language and culture learning. Essential Teacher, 29-31.

Reinders, H. (2011). Digital Storytelling in the Foreign Language Classroom. ELTWorldOnline.com, 3.

Robin, B. R. (2008). Digital Storytelling: A Powerful Techology Tool for the 21st Century Classroom. Theory into Practice, 220-228.

Robin, B. R. (2016). The Power of Digital Storytelling to Support Teaching and Learning. Digital Education Review, 30, 17-29.

Roy, C. K. (2016). Be Creative and Collaborative: Strategies and Implications of Blogging in EFL Classes. English Language Teaching, 9(7), 129-145.

Tompkins, G. (1982). Seven reasons why children should write stories. Language Arts, 718-721.

Umrani, W., Ahmed, U., \& Memon, P. (2015). Examining the absorptive capacity construct: A validation study in the Pakistani banking context. Management Science Letters, 5(12), 1053-1058.

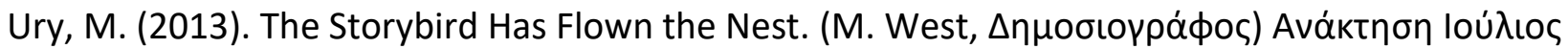
2019, aró http://theliteraryplatform.com/magazine/2013/03/the-storybird-has-flownthe-nest/

Vasudevan , L., DeJaynes, T., \& Schmier, S. (2010). Multimodal pedagogies: Playing, teaching, and

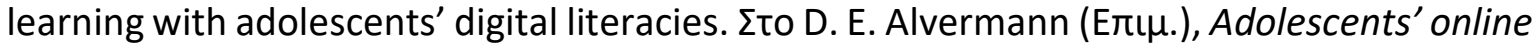

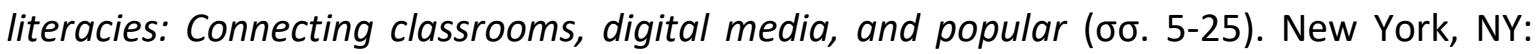
Peter Lang. 
Xu, Y., Park, H., \& Baek, Y. (2011). A New Approach Toward Digital Storytelling: An Activity Focused on Writing Self-efficacy in a Virtual Learning Environment. Educational Technology \& Society, 14(4), 181-191.

Zin, M. L. M., \& Ibrahim, H. (2020). The Influence of Entrepreneurial Supports on Business Performance among Rural Entrepreneurs. Annals of Contemporary Developments in Management \& HR (ACDMHR), 2(1), 31-41.

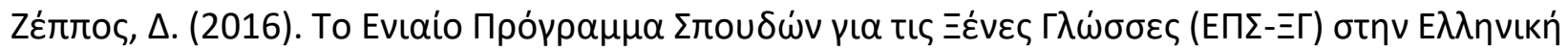

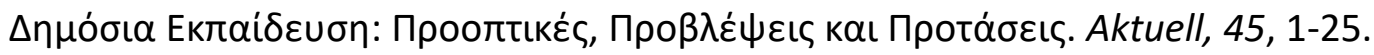

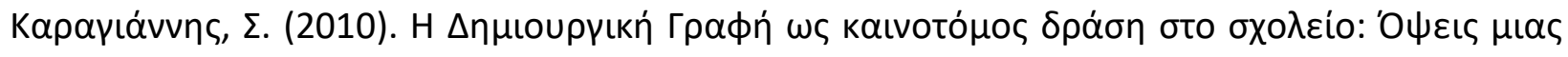

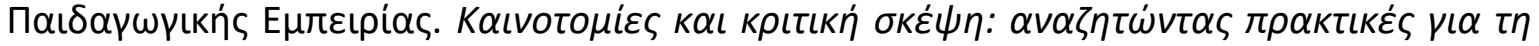

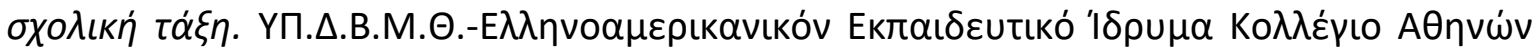

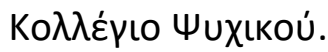

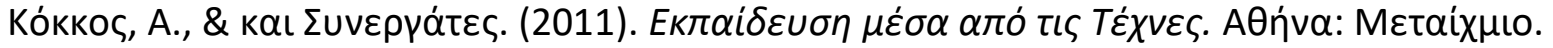

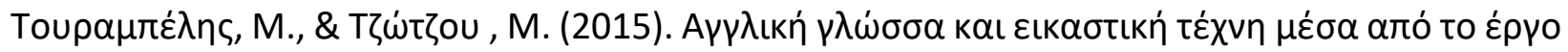

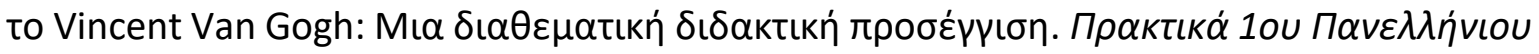

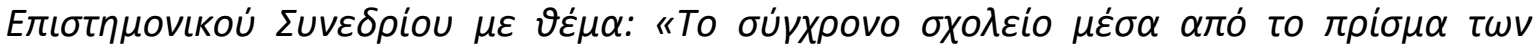

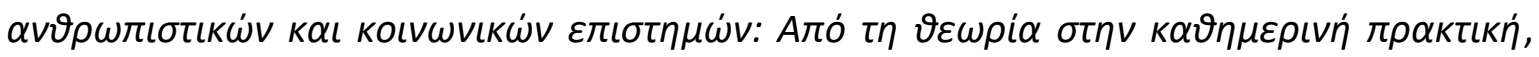

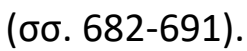

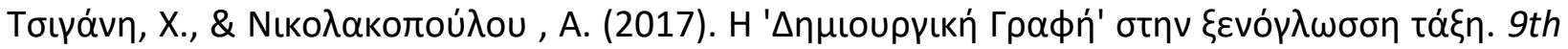
Conference on Informatics in Education, ( $\sigma \sigma .325-336)$.

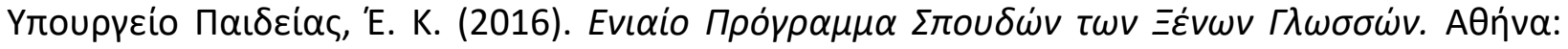

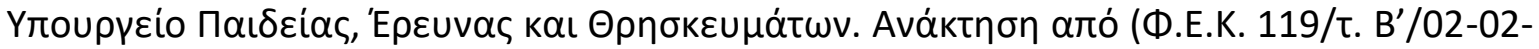
2006). 